\title{
Coleção de simuliídeos (Diptera - Simuliidae)de Adolpho Lutz, sua história e importância
}

\author{
Adolpho Lutz's collection of black \\ flies (Diptera - Simuliidae), its \\ bistory and importance
}

\author{
Ana Margarida Ribeiro do Amaral-Calvão \\ Mestranda do Programa de Pós-Graduação da Universidade \\ Rural do Rio de Janeiro (UFRRJ) \\ calvao@ioc.fiocruz.br \\ Marilza Maia-Herzog \\ Pesquisadora titular do Departamento de Entomologia/IOC/Fiocruz \\ Doutora em biologia parasitária \\ Av. Brasil, 4365 \\ 21045-900 Rio de Janeiro — RJ Brasil \\ mherzog@ioc.fiocruz.br
}

\begin{abstract}
O presente trabalho é parte de uma dissertação de mestrado em elaboração no curso de pós-graduação em biologia animal da Universidade Federal do Rio de Janeiro (UFRJ), apoiado pela CAPES. Nele, são apresentadas as espécies de simuliídeos que pertencem à coleção de Adolpho Lutz depositadas no Laboratório de Simuliídeos e Oncocercose do Departamento de Entomologia do Instituto Oswaldo Cruz (IOC). Pioneiro no estudo desses dípteros, Lutz descreveu cerca de 25 espécies de diferentes localidades do Brasil. Vetor da oncocercose, os simuliídeos tiveram sua importância médico-sanitária reconhecida em fins da década de 1920.

PALAVRAS-CHAVE: simuliídeo, oncocercose, insetos, díptero, Adolpho Lutz.

This paper is part of a master's thesis currently being written under the auspices of the Post-Graduate Program in Animal Biology of the Federal University of Rio de Janeiro, with support from CAPES. It presents the species of black flies in Adolpho Lutz's collection, beld at the Laboratory of Black Flies and Oncocercosis of the Department of Entomology of the Instituto Oswaldo Cruz. A pioneer in the study of these dipterons, Lutz described about 25 species from different places in Brazil. A vector of round worm, the black fly's importance to public health was recognized at the end of the 1920 s.
\end{abstract}

KEYWORDS: black fly, round worm, insects, dipteron, Adolpho Lutz.

\section{Introdução}

S simuliídeos são dípteros nematóceros, vulgarmente conhecidos no Brasil como borrachudos, piuns ou casaquinhos-de-couro; nos países de língua hispânica, jejenes, e nos de língua inglesa, black flies. A família Simuliidae possui ampla distribuição geográfica e grande importância médico-veterinária, pois inclui espécies vetoras de agentes etiológicos de doenças, tanto para o homem como para outros animais. 
Após a estação chuvosa, as populações de simulíideos atingem grandes números em áreas propícias às espécies antropofílicas, e suas picadas ocasionam um "ataque-incômodo". A hematofilia dos simuliídeos acarreta sérios prejuízos econômicos, afetando, principalmente, a agropecuária e o turismo.

A coleção de simuliídeos de Adolpho Lutz pertence ao acervo da Coleção Entomológica do Instituto Oswaldo Cruz (IOC), e encontrase sob a guarda do Laboratório de Simuliídeos e Oncocercose do Departamento de Entomologia da mesma instituição. Formada entre 1903 e 1925, a coleção possui larvas, pupas, machos e fêmeas coletados em diferentes localidades do Brasil, principalmente na região Sudeste, destacando-se a serra da Bocaina, em São Paulo. O material está conservado de quatro maneiras: sob lâmina e lamínula, em "ampolas" (figs. 1), em álcool (fig.2), ou alfinetados (figs. 3 e 4).

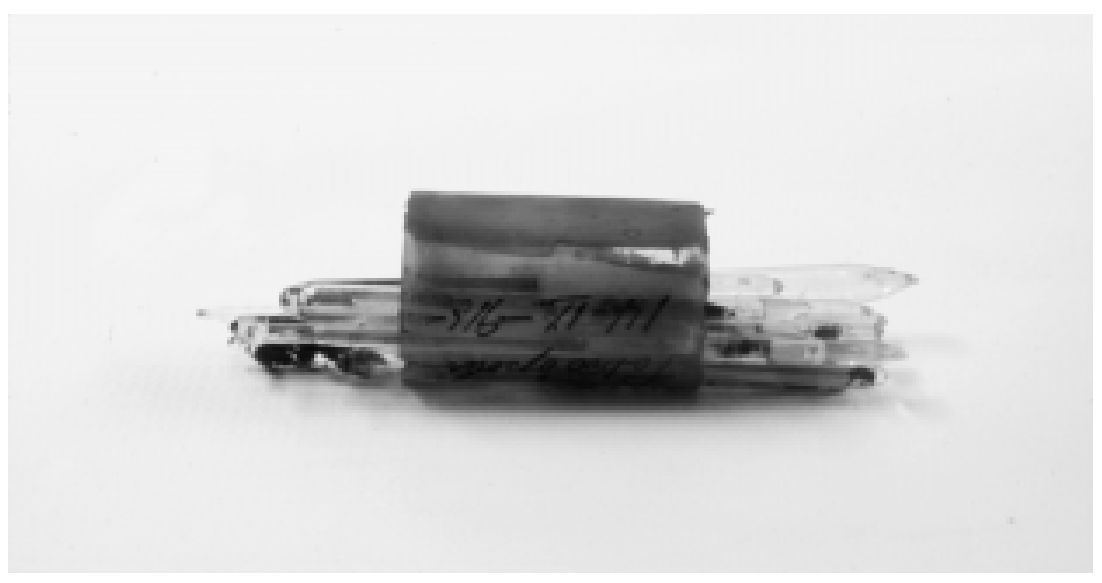

Fig. 1 - Exemplares da coleção de simuliídeos de Adolpho Lutz conservados em ampolas de vidro.

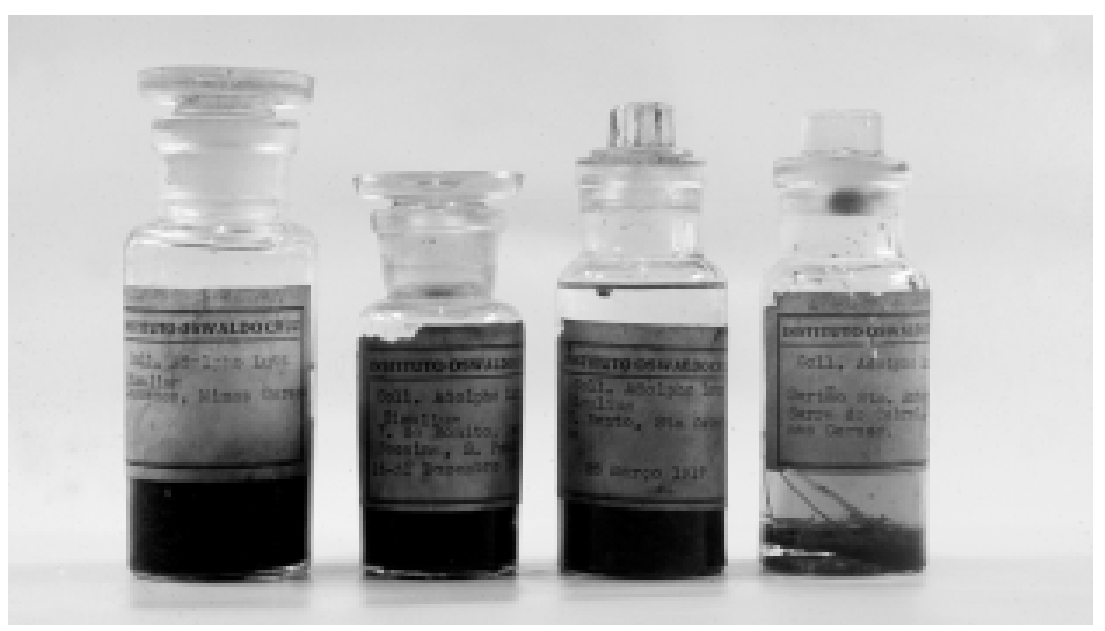

Fig. 2 - Exemplares da coleção de simuliídeos de Adolpho Lutz conservados em álcool. 


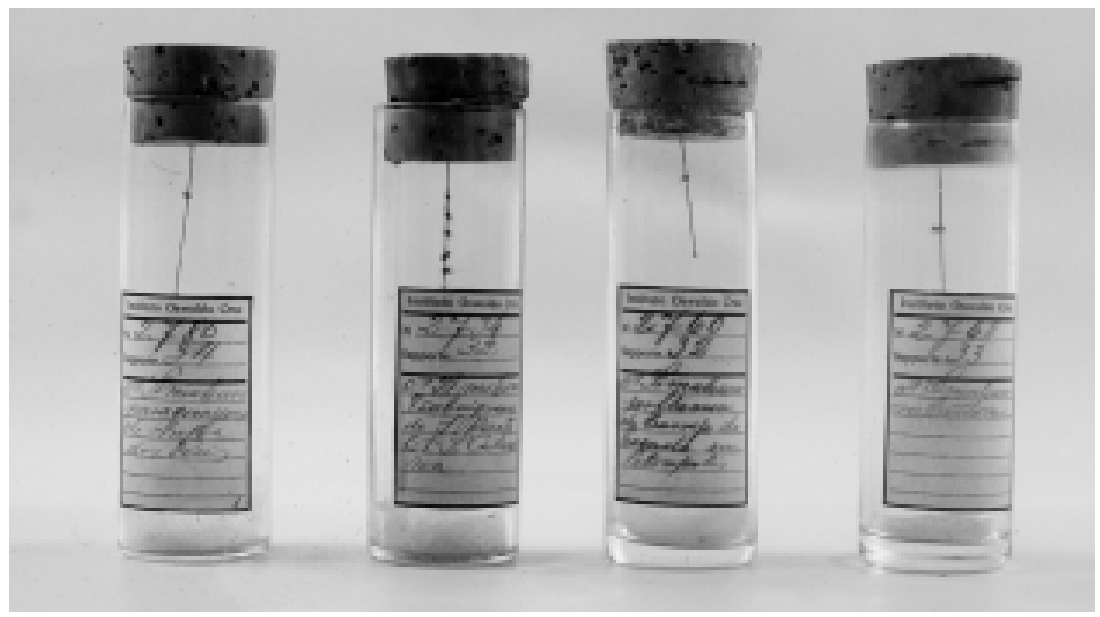

Fig. 3 - Exemplares da coleção de simuliídeos de Adolpho Lutz alfinetados.

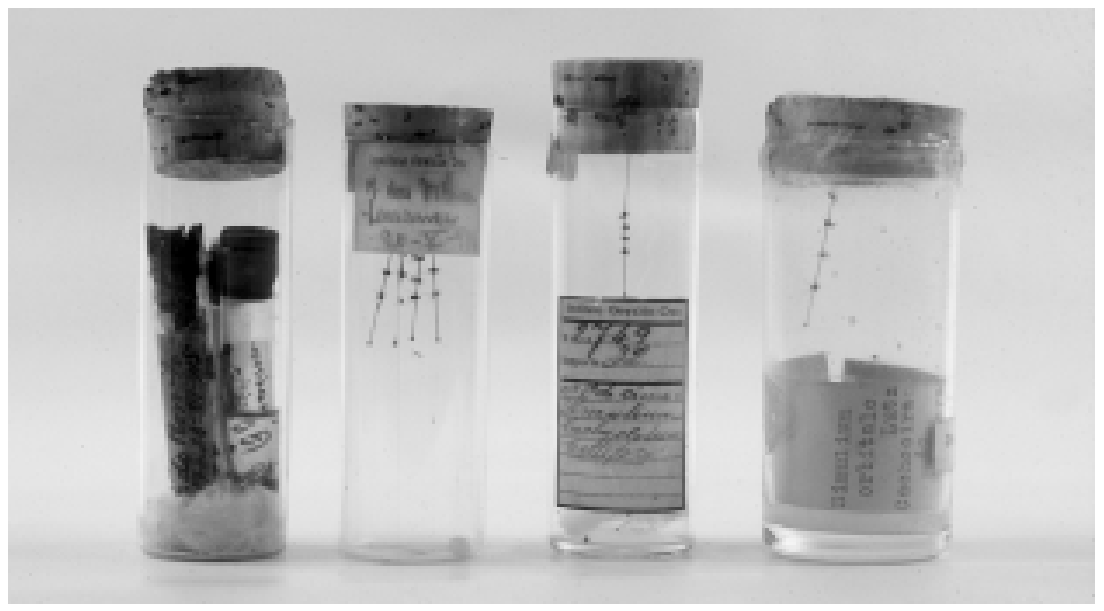

Fig. 4 - Detalhe dos exemplares da coleção de simulíídeos de Adolpho Lutz.

Em trabalho publicado em 1903 sobre insetos sugadores de sangue observados no Rio de Janeiro e em São Paulo, Lutz (1903) chamou a atenção para o pouco conhecimento que se tinha sobre o grupo, mencionando somente quatro espécies conhecidas e apenas uma descrita cientificamente. Esta era a Simulium nigrimanum descrita por Macquart em 1838. Em 1905, Emílio Goeldi descreveu uma segunda espécie, a Simulium amazonicum.

Lutz passou a estudar os simuliídeos efetivamente alguns anos depois, e em 1909 publicou o primeiro trabalho concernente ao assunto, tratando de algumas espécies que ocorrem no Brasil. Lutz mencionou então 12 espécies e descreveu cinco, nativas: Simulium rubrithorax Lutz, 1909, S. scutistriatum Lutz, 1909, Lutzsimulium hirticosta Lutz, 1909, S. exiguum Roubaud, 1906 (na realidade tratava-se de S. lutzi Knab, 1913) e $S$. varians Lutz. No referido trabalho, além de fornecer dados que 
caracterizam a família Simuliidae quanto à morfologia geral, ressaltou aspectos relevantes da fisiologia e biologia desses dípteros, e elaborou uma chave de classificação para as espécies mencionadas. Relatou, ainda, a ocorrência de casos fatais entre animais e humanos atacados por enxames colossais de simulíideos na Hungria e nos Estados Unidos (Lutz, 1909).

Sentindo necessidade de conhecer melhor o grupo, Lutz passou a arregimentar coletores e cientistas amigos para obter espécimes. Entre os colaboradores no Brasil, destacamos Oswaldo Cruz, Carlos Chagas, Parreiras Horta, Gurgel do Amaral, Arthur Neiva, David Madeira, Von Luetzelburg e J. G. Foetterle. Lutz também manteve intensa troca de materiais e informações com pesquisadores de outros países, e remeteu exemplares de simuliídeos para coleções da Alemanha, Grã-Bretanha e Estados Unidos. Apesar da extensão alcançada por essa rede, a maioria dos exemplares foi coletada pelo próprio Lutz em excursões a arrabaldes do Rio de Janeiro e regiões vizinhas.

Em 1910, publicou trabalho fornecendo novas informações fisiológicas e, principalmente, morfológicas. Redescreveu as espécies S. rubrithorax, L. hirticosta, S. pertinax Kollar, 1832; S. perflavum Roubaud, 1906; S. exiguum, S. varians, S. paraguayense Schrottky, 1909; e ainda a espécie S. pernigrum (Lutz, 1910) atualmente pertencendo ao gênero Lutzsimulium pernigrum. O cientista descreveu ainda as espécies S. orbitale Lutz, 1910; S. infuscatum Lutz, 1909 (na realidade trata-se de S. pertinax), S. subnigrum Lutz, 1910; S. distinctum Lutz, 1910; S. incrustatum Lutz, 1910; e S. auristriatum Lutz, 1910.

Em 1917, Adolpho Lutz publicou outro trabalho sobre o grupo, redescrevendo a espécie $S$. amazonicum, que havia sido descrita por Goeldi em 1905.

Em viagem científica realizada de março a outubro de 1912, com Astrogildo Machado, pelo rio São Francisco e alguns de seus afluentes, a convite da Inspetoria de Obras contra a Seca, Lutz coletou numerosos insetos, inclusive simuliídeos, em sua maioria formas imaturas obtidas nos criadouros. No relatório da viagem, publicado em 1915, registrou a grande capacidade de dispersão dos simuliídeos através do vôo, tirando proveito dos ventos e da umidade do ar. As espécies que coletou foram: S. brevibranchium n. sp. Lutz e Machado, 1915 (sinonímia de S. brachycladum, Lutz, 1910); S. diversifurcatum Lutz, 1910 (sinonímia de S. subnigrum); S. incrustatum, S. orbitale, S. paraguayense, S. pruinosum Lutz, 1910; (S. pruinosum é sinonímia de S. nigrimanum Macquart, 1838); S. rubrithorax, S. spinibranchium Lutz, 1910; e S. subviride Lutz e Machado, 1915 (S. subviride é sinonímia de S. subpallidum Lutz, 1910) (Crosskey e Howard, 1996).

Em outra viagem científica, desta vez à Argentina, ao Uruguai e ao Paraguai, entre janeiro e março de 1918, em companhia de Heráclides 
César de Souza Araújo e Olympio da Fonseca Filho, Adolpho Lutz coletou mais exemplares de simuliídeos, entre eles S. pertinax, S. orbitale, S. amazonicum, S. incrustatum, S. subviridee S. rubrithorax. No trabalho publicado em 1918, redescreveu três espécies: S. paraguayense Schrottky, 1909, S. inexorabile Schrottky, 1909 [sinonímia de S. pertinax (Crosskey e Howard, 1996)] e S. paranaense Schrottky, 1909.

É importante ressaltar a acuidade das observações feitas pelo cientista, que, já naquela época, chamava a atenção para as modificações morfológicas sofridas no padrão de desenhos do escudo dos simuliídeos de acordo com a incidência da luz, e o estado de conservação dos exemplares observados. Relatou as mudanças observadas na coloração do corpo desses dípteros devido ao depósito de pigmento nos tecidos após a hemólise da hemoglobina contida no sangue ingurgitado pelas fêmeas. O aparelho bucal de nematóceros hematófagos foi descrito por Lutz em 1912. Neste artigo, observou que machos do gênero Simulium apresentam todas as partes bucais apesar de não se alimentarem de sangue, e descreveu parcialmente a postura de ovos de algumas espécies indígenas de Simulium.

Segundo Crosskey e Howard (1996), existem atualmente 1.663 espécies de simuliídeos cientificamente nomeadas, com cerca de seiscentas sinonímias. No Brasil até 1967 tinham sido catalogadas, aproximadamente, 55 espécies (Vulcano, 1967), dessas, 25 descritas por Lutz. Crosskey e Howard (1996) e Crosskey (2002) referem-se a cerca de 83 espécies de simuliídeos descritas no país.

Ainda hoje há controvérsias quanto à situação taxonômica de várias espécies descritas no Brasil, pois o grupo apresenta características morfológicas homogêneas. Shelley et al. $(1987,1982)$ suspeitam da existência de complexos de espécies entre os simuliídeos no país. São escassas as descrições apropriadas, as chaves viáveis para identificação e monografias sobre o assunto. Para uma compreensão mais completa empregam-se, hoje, conhecimentos em âmbito citogenético, microscopia mais refinada e associação com a biologia de cada espécie (MaiaHerzog, 1991).

Trabalhos com a Coleção de Adolpho Lutz já foram realizados por Vulcano, Py-Daniel, Hamada, Shelley, Coscarón e ainda MaiaHerzog (1991) e Maia-Herzog et al. (1985).

Lutz prosseguiu os estudos sobre insetos nematóceros, publicando, em 1922, mais um artigo dedicado a estes dípteros. Dedica aí grande espaço aos simulíídeos, fornecendo dados sobre sua taxonomia, morfologia e biologia. Ainda não se havia provado o papel destes insetos como vetores de doença humana, mas Lutz (op. cit.) levantava essa hipótese, visto que as fêmeas se alimentam em intervalos bastante longos, favorecendo o desenvolvimento de parasitos.

Não se deve omitir sua contribuição ao conhecimento da simuliofauna de outros países da América do Sul. Em importante trabalho sobre dípteros hematófagos na Venezuela, publicado em 1928, apresentou 
algumas redescrições e descreveu duas espécies novas: $S$. lugubre Lutz e Nuñes Továr, 1928 (sinonímia de S. mexicanum Bellardi, 1862) e $S$. versicolor Lutz e Nuñes Továr, 1928 (sinonímia de S. metallicum Bellardi, 1859). Neste trabalho, analisou o método utilizado para conservar e observar estes dípteros e produzir estampas representando-os.

Em artigo dedicado à biologia de ambientes lóticos (Lutz, 1930) tratou mais uma vez dos simuliídeos, dando ênfase aos aspectos biológicos. No trabalho sobre a transmissão de doenças através de "sugadores de sangue", mencionou a participação das espécies antropofílicas como vetoras da "filariose" humana causada por Onchocerca (Lutz, 1936). ${ }^{1}$

César Pinto deu continuidade aos trabalhos de Lutz publicando artigo dedicado exclusivamente aos simuliídeos em 1930, no qual relatou aspectos de sua biologia e morfologia, fazendo comentários sobre o órgão descoberto por Lutz no terceiro segmento dos palpos maxilares. Pinto reeditou, neste trabalho, a chave para a classificação das fêmeas de Simulium existentes no Brasil publicada por Lutz em 1910, e mencionou importante trabalho de Breadbane Blacklock publicado em 1926 sobre a transmissão de Onchocerca volvulus pela picada de Simulium damnosum Theobald. Apresentou, também, a distribuição geográfica dos simuliídeos na região neotropical (Argentina, Brasil, Chile, México, Paraguai e Venezuela). O artigo é, na verdade, uma súmula de informações obtidas anteriormente por Lutz.

Em 1932, Pinto publicou trabalho mais detalhado e importante sobre as espécies de simuliídeos da América Central e do Sul, registrando que totalizavam "cento e poucas espécies, sendo o Brasil o país onde melhor foram estudados, graças aos trabalhos básicos de Adolpho Lutz, realizados durante muitos anos e firmados em estupendo material colecionado por este eminentíssimo sábio dotado de invulgar capacidade e invejável tenacidade ao estudo da zoologia e parasitologia sul-americanas".

Das 36 espécies encontradas no Brasil, 25 tinham sido descritas por Lutz, dez por outros pesquisadores, e uma por Lutz e Pinto. Hoje se sabe que entre elas encontram-se seis sinonímias (o S. pruinosum, por exemplo, é sinonímia de $S$. nigrimanum).

César Pinto continuou debruçado sobre a família Simuliidae nos trabalhos publicados em 1936 e 1940, cedendo o bastão, de 1940 a

\footnotetext{
${ }^{1}$ A confirmação dos simuliídeos como vetores da Onchocerca volvulus se deu em 1926, em Serra Leoa, pelo médico inglês B. Backlock. O primeiro caso de oncocercose reportado e confirmado no Brasil foi descrito por Bearzoti et al. (1967). Mas os estudos sobre simuliídeos só passaram a ter maior importância médica após a confirmação de um foco amazônico da doença em Roraima, por Moraes e Chaves (1974). Em 1986, foi descrito o primeiro caso autóctone de oncocercose fora do foco amazônico, procedente de Goiás (Gerais e Ribeiro, 1986). Maia-Herzog (1999) relata o histórico da oncocercose no Brasil e a distribuição e expansão da doença no país através dos movimentos migratórios de garimpeiros.
} 
1950, a. Vulcano e D'Andretta Jr., que redescreveram algumas das espécies de Lutz.

\section{Levantamento dos exemplares depositados na Coleção de Simuliídeos de Adolpho Lutz}

A Coleção de Lutz possui material coletado em diferentes localidades do Brasil, principalmente na região Sudeste, destacando-se a Fazenda do Bonito, de propriedade de um dos irmãos de Adolpho Lutz, na serra da Bocaina, em São Paulo, de onde provêm muitos dos exemplarestipo das espécies descritas por Lutz.

O material, coletado no período de 1903 a 1925, contém aproximadamente 1.400 exemplares distribuídos em dois gêneros (Simuliume Lutzsimulium), 12 subgêneros (Chirostilbia, Hellichiella, Hemicnetha, Inaequalium, Kempfsimulium, Latreille, Notolepria, Psaroniocompsa, Psilopelmia, Pternaspatha, Trichodagmia e Wtzsimulium), e 41 espécies. Destes, 518 exemplares encontram-se conservados sob lâmina/lamínula (dois gêneros e nove subgêneros), distribuídos em 29 espécies, 18 tipos, entre três holótipos; 830 exemplares alfinetados (dois gêneros e 12 subgêneros), sendo 48 tipos, distribuídos em 35 espécies; totalizando 51 exemplares-tipo, destes, sete holótipos, distribuídos em 35 espécies.

Em 1909, Lutz citou S. montanum Philippi, 1865, e traduziu a descrição original feita em língua alemã por Philippi num catálogo de dípteros chilenos. S. montanum teria sido encontrada picando o homem na serra da Bocaina, a 1.500 metros acima do nível do mar. Talvez por achar estranho o fato de esta espécie ter sido coletada no Brasil, Lutz declarou que se esta fosse idêntica à espécie de Philippi deveria ter transposto os Andes, algo excepcional, que talvez se explicasse pela facilidade com que a mesma se criava em lugares elevados.

Porém, em 1910, Lutz restituiu ao material identificado como $S$. montanum o nome de S. pernigrum (atualmente Lutzsimulium pernigrum), visto que sua identidade com a espécie chilena era bastante duvidosa, acrescentando apenas poucos detalhes à descrição. O material reunido era composto por um grande número de fêmeas realizando repasto sanguíneo na barriga de cavalos, e em larvas e pupas provenientes da serra da Bocaina.

Pinto $(1932,1930)$ mencionou, de forma breve, S. pernigrum, citando a localidade da espécie descrita por Philippi (montanhas de Chacabuco, perto de Catemu, no Chile), mas também se referiu a $S$. montanum Kertész, 1902.

Edwards (1931) colocou S. montanum no subgênero Cnephia. Mais tarde, Smart (1945) transferiu a espécie para Cnephia montanum. Porém Wygodzinsky e Coscarón (1973) passaram a espécie ao gênero Araucnephia montana (Philippi) (Coscarón, 1991). 
D'Andretta e D'Andretta (1952) transferiram a espécie de gênero e redescreveram Lutzsimulium pernigrum, tendo como base material de larvas e pupas capturadas entre 1.800 e 2.200 metros de altitude e proveniente de Itatiaia, Rio de Janeiro. Em 1962, Vulcano fez nova menção à $L$. pernigrum, desta vez com exemplares coletados em diversos pontos da localidade tipo, e comentou o fato de a espécie ter sido originalmente descrita como $S$. montanum.

Não tivemos como acessar os catálogos aos quais Lutz e Pinto se referem, não encontrando, na literatura, nenhuma menção ao $S$. montanum descrito no Chile por Philippi, em 1865. Os catálogos de Crosskey e Howard (1996) e Vulcano (1967) não mencionam qual seria a espécie que estaria em sinonímia com S. montanum Philippi, 1865.

Os exemplares de $S$. annulatum Philippi, 1865, encontrados na coleção de simuliídeos de Lutz não possuem nenhuma informação sobre sua origem. A literatura, até o momento, só menciona esta espécie em localidades do Chile e Argentina. Caso seja confirmada a identificação, podem ser exemplares coletados por Lutz na expedição científica ao rio Paraná e a Assunção, com volta por Buenos Aires, Montevidéu e Rio Grande. O material pode ainda ter sido enviado a Lutz por algum pesquisador.

S. clavigerum não consta na literatura. Trata-se de uma simples sinonímia ou de uma espécie válida, que Lutz nomeou mas não teve tempo de descrever. Acreditamos que seja mais provável a primeira hipótese, já que as localidades de coleta são Petrópolis e serra da Bocaina, duas regiões com grande histórico de estudos no que se refere aos simuliídeos.

Do material depositado entre lâmina/lamínula, podemos destacar S. quinquefurcatum. Apesar de possuirmos apenas uma lâmina de filamentos respiratórios da pupa, acreditamos ser uma espécie válida devido à forma distinta e disposição dos ramos destes filamentos. No entanto, não podemos sustentar tal afirmação com apenas um exemplar de parte de um imaturo.

S. latipes (Meigen, 1804) (International Commission on Zoological Nomenclature), e que, segundo Crosskey e Howard (1996), é holótipo válido na opinião da ICZN, 1416, de procedência alemã. O exemplar contido na coleção de Lutz consta como sintipo, proveniente da localidade de Rio Grande, Minas Gerais, em 23.9.1903.

O levantamento da coleção resultou na elaboração de uma chave simplificada das espécies do Brasil descritas por Lutz. Foi elaborada uma tabela de distribuição destas espécies (tabelas 1 e 2) a partir dos dados obtidos através do levantamento da coleção de simuliídeos de Adolpho Lutz e de dados levantados na Coleção de Referência do Laboratório de Simuliídeos e Oncocercose, cujo patrimônio inclui exemplares obtidos em diversas localidades do Brasil, com amostras significativas de todas as suas regiões. 
Tabela I: total de exemplares na coleção de alfinetados de simuliídeos de Adolpho Lutz

\begin{tabular}{|c|c|c|c|c|c|c|}
\hline Lâmina/espécie & Larva & Pupa & Fêmea & Macho & Exemplares duvidosos & Total \\
\hline S. amazonicum & - & - & 31 & 1 & 13 adultos +2 desaparecidos* & 45 \\
\hline S. annulatum ${ }^{1}$ & - & - & 1 & - & - & 1 \\
\hline S. auristriatum & - & - & 22 & 2 & 4 & 28 \\
\hline S. botulibranchium & - & - & 18 & 2 & 2 adultos +4 pupas & 26 \\
\hline S. clavibranchium & - & 5 & 22 & 12 & 9 adultos & 48 \\
\hline S. clavigerum ${ }^{2}$ & - & - & 1 & 1 & 2 adultos & 4 \\
\hline S. distinctum & - & - & 68 & - & 7 adultos & 75 \\
\hline S. diversibranchium & - & - & 3 & 3 & 1 adulto & 7 \\
\hline $\begin{array}{l}\text { S. diversifurcatum = } \\
\text { S. subnigrum }\end{array}$ & - & - & 27 & 8 & 1 adulto & 36 \\
\hline S. exiguum & - & - & 3 & - & - & 3 \\
\hline L. flavopubescens & - & - & 2 & - & - & 2 \\
\hline L. hirticosta & - & - & 4 & 4 & 1 adulto +1 desaparecido* & 9 \\
\hline $\begin{array}{l}\text { S. incrustatum }= \\
\text { S. pertinax }\end{array}$ & - & - & 3 & - & - & 3 \\
\hline S. infuscatum & - & - & 3 & - & 2 adultos & 5 \\
\hline S. latipes ${ }^{3}$ & - & - & - & - & 1 adulto & 1 \\
\hline S. lutzi & - & - & 1 & - & - & 1 \\
\hline S. minusculum & - & - & 5 & 1 & - & 6 \\
\hline $\begin{array}{l}\text { S. } \text { montanum }= \\
\text { S. } \text { argyreatum }^{4}\end{array}$ & - & - & 8 & - & - & 8 \\
\hline S. nigrimanum & - & - & 12 & 1 & - & 13 \\
\hline S. obesum & - & - & 13 & - & - & 13 \\
\hline S. paraguayense & - & 1 & 25 & 2 & 4 adultos +1 desaparecido* & 32 \\
\hline S. perflavum & - & - & 13 & 9 & 2 adultos & 24 \\
\hline L. pernigrum & - & - & 33 & 4 & 1 adulto & 38 \\
\hline S. pertinax & - & - & 88 & 36 & 13 adultos + 1 desaparecido* & 137 \\
\hline S. rorotaense & - & - & 1 & - & - & 1 \\
\hline S.rubrithorax & - & 8 & 20 & - & 3 desaparecidos* & 28 \\
\hline S. scutistriatum & - & - & 2 & - & - & 02 \\
\hline L. simplicicolor & - & - & 88 & - & 06 adultos & 94 \\
\hline S. spinibranchium & - & - & 17 & - & 1 desaparecido* & 17 \\
\hline S. subnigrum & - & - & 10 & - & 2 adultos & 12 \\
\hline S. subpallidum & - & 3 & 25 & - & 4 adultos & 32 \\
\hline $\begin{array}{l}\text { S. subviride = } \\
\text { S. subpallidum }\end{array}$ & - & 5 & 23 & - & 5 adultos & 33 \\
\hline S. varians & - & - & 26 & 4 & 3 adultos +1 desaparecido* & 33 \\
\hline Não identificados & - & - & 12 & - & 1 adulto & 13 \\
\hline Total geral & - & 22 & 630 & 90 & $\begin{array}{c}88 \text { (84 adultos e } 4 \text { pupas) } \\
+10 *\end{array}$ & 830 \\
\hline
\end{tabular}

${ }^{1}$ S. annulatum: não constam data, localidade e coletores. Ver discussão.

${ }^{2}$ S. clavigerum: provável tipo ou sinonímia de outra espécie, proveniente de Petrópolis em 29 e 30.x.1912 e da Fazenda do Bonito, serra da Bocaina de 1 a 10.2.1917. Macho completo. Ver discussão.

${ }^{3}$ S. latipes: (Meigen, 1804), nome preocc. Na coleção de Lutz está como sintipo, localidade de R. Grande, Minas Gerais em 23.9.1903. Ver discussão.

${ }^{4}$ S. montanum: sem data, localidade e coletor. Ver discussão.

* Exemplares desaparecidos —, não foram contados. 
Tabela 2: total de exemplares na coleção de lâminas de simulídeos de Adolpho Lutz

\begin{tabular}{|c|c|c|c|c|c|c|}
\hline Lâmina / espécie & Larva & Pupa & Fêmea & Macho & Exemplares duvidosos & Total \\
\hline S. amazonicum & 1 & 11 & 10 & 1 & - & 23 \\
\hline S. auristriatum & 4 & 12 & 4 & 3 & 4 adultos & 27 \\
\hline S. botulibranchium & 1 & 3 & - & 1 & - & 5 \\
\hline S. brachycladum & - & 6 & - & - & - & 6 \\
\hline S. brevifurcatum & - & 30 & 1 & 1 & - & 32 \\
\hline S. clavibranchium & 1 & 13 & - & 3 & 1 ampola c/ ? exemplares* & 17 \\
\hline S. distinctum & - & 5 & 7 & 1 & - & 13 \\
\hline S. diversibranchium & - & 7 & - & - & 1 adulto & 8 \\
\hline S. exiguum & - & - & 3 & - & $\begin{array}{c}1 \text { lâmina desaparecida c/ ? } \\
\text { exemplares* }\end{array}$ & 3 \\
\hline L. flavopubescens & 4 & 6 & - & - & 2 adultos & 12 \\
\hline L. hirticosta & 2 & 9 & 1 & 1 & - & 13 \\
\hline S. incrustatum & 2 & 33 & 3 & 3 & 17 adultos & 58 \\
\hline S. mexicanum & 1 & 3 & - & - & - & 4 \\
\hline S. nigrimanum & - & 7 & 7 & 3 & 8 lâminas c/ ? exemplares* & 17 \\
\hline S. obesum & - & 4 & 2 & - & - & 6 \\
\hline S. orbitale & - & 1 & 3 & - & - & 4 \\
\hline S. ornatumi & - & - & - & - & 2 lâminas c/ ? exemplares* & $?$ \\
\hline S. paraguayense & - & 6 & 6 & - & 4 lâminas c/? exemplares* & 12 \\
\hline S. perflavum & 1 & 5 & - & 1 & $\begin{array}{l}1 \text { ampola c/ ? exemplares* + } \\
06 \text { Lâminas c/ ? exemplares* }\end{array}$ & 7 \\
\hline L. pernigrum & - & 8 & - & - & - & 8 \\
\hline S. pertinax & - & 56 & 12 & 11 & $\begin{array}{l}3 \text { ampolas c/ ? exemplares* } \\
+2 \text { Lâminas desaparecidas* }\end{array}$ & 79 \\
\hline S. quinquefurcatum sp & - & 1 & - & - & $1^{1}$ & 2 \\
\hline S.rubrithorax & 9 & 19 & 5 & - & - & 33 \\
\hline L. simplicicolor & - & 3 & 3 & - & - & 6 \\
\hline S. subclavibranchium & 2 & 3 & - & - & - & 5 \\
\hline S. subnigrum & - & 43 & 3 & 1 & $\begin{array}{l}1 \text { ampola desaparecida* }+ \\
1 \text { adulto }\end{array}$ & 48 \\
\hline S. subpallidum & - & 50 & 1 & 1 & - & 52 \\
\hline Total Geral & 28 & 344 & 71 & 31 & 26 adultos $+29 *$ & 500 \\
\hline
\end{tabular}

*Lâminas e ampolas, que se encontram em péssimo estado de conservação, não havendo como observar quantos e quais os estágios dos exemplares de simuliídeos estão nelas contidos; e as desaparecidas, não puderam ser somadas ao resultado final. Somente os adultos foram somados ao total geral de exemplares contidos na coleção de lâminas de simuliídeos de Adolpho Lutz.

${ }^{1}$ Exemplar identificado como S. quinquefurcatum, mas diferente do exemplar contido na outra lâmina.

\section{Conclusão}

Não deixa de ser surpreendente o fato de Lutz conseguir tempo para observações tão peculiares e detalhadas dos simuliídeos, quando se sabe que, concomitantemente, desenvolvia numerosos trabalhos sobre temas muito distintos. As lâminas das coleções de Lutz e Pinto foram montadas no início do século XX com técnicas e equipamentos rudimentares. Além disso, muitas estão malconservadas, impregnadas por fungos, o que prejudica e/ou impossibilita a observação do material. Apesar disso, podemos afirmar que os artigos de Lutz ainda representam as contribuições mais importantes para o conhecimento dos simuliídeos no Brasil. 


\section{REFERÊNCIAS BIBLIOGRÁFICAS}

Anderson, J. R. e Voskuil, G. B. A. 1963

Araújo-Coutinho, C. J. P. C.

Bearzoti, P.; Lane, E. e Menezes Jr., J. 1967

Calvão-Brito, R. H. S. e Maia-Herzog, M. 1988

Carvalho, M. S.; Pina, M. de F. de e Santos, S. M. dos 2000

Crosskey, R. W. 2002

\section{Crosskey, R. W. e} Howard, T. M. 1996

Gerais, B. B. e Ribeiro, T. C. 1986

Lutz, A. e Castro, G. de O.

1936

Lutz, A.

Lutz, A.; Souza Araujo, H. C. e Fonseca Filho, O. da 1918

Lutz, A.

Lutz, A. e Machado, A. 1915

Lutz, A. 1912

Lutz, A.

1910
'Reduction in milk production caused by feeding of black flies (Diptera: Simuliidae) on dairy cattle in California, with notes on feeding activity on other animals'. Mosquito News, vol. 23, no 2, pp. 126-31.

Abundância sazonal da população de formas imaturas de Simulium pertinax Kollar, 1832 (Diptera Simuliidae) e da entomofauna associada, no município de Paraty, RJ. Dissertação de mestrado, Rio de Janeiro, Instituto de Biologia, Universidade Federal Rural do Rio de Janeiro, IV + 81p.

'Relato de um caso de oncocercose adquirido no Brasil'. Rev. Paulista de Med., 70: 102.

'Modificação na técnica para montagem de simulídeos em lâmina/lamínula'. Rev. Brasileira de Zoologia. (no prelo)

Conceitos básicos de sistemas de informação geográfica e cartográfica aplicados à saúde. Rede Interagencial de Informações para Saúde (RIPSA). Brasília, Organização Pan-Americana de Saúde/Ministério da Saúde, 122p.

Second update to the taxonomic and geographical inventory of world blackflies (Diptera: Simuliidae) Londres, Department of Entomology, The Natural History Museum.

A new taxonomic and geographical inventory of world blackflies (Diptera: Simuliidae).

Londres, Department of Entomology, The Natural History Museum.

'Relatos de casos - oncocercose: primeiro caso autóctone da região centro-oeste do Brasil'. Revista da Sociedade Brasileira de Medicina Tropical, vol. $19, \mathrm{n}^{\circ}$ 2, pp. 105-7.

'Considerações sobre a transmissão de doenças por sugadores de sangue'. A Folha Medica, Rio de Janeiro, 17 (21): 320-8.

Estudios de zoologia e parasitologia venezolanas. Rio de Janeiro, 133p., 26 estampas.

'Viagem scientifica no rio Paraná e a Assuncion com volta por Buenos Ayres, Montevideo e Rio Grande'. Mem Inst. Oswaldo Cruz, 10 (2): 104-73, estampas 20-75.

'Terceira contribuição para o conhecimento das espécies brasileiras do gênero Simulium. O pium do norte (Simulium amazonicum)'.

Rio de Janeiro, Instituto Oswaldo Cruz. Separata de Mem. do Inst. Oswaldo Cruz, Rio de Janeiro, 9(1): 63-7, estampas 25.

'Viagem pelo rio S. Francisco e por alguns de seus afluentes entre Perapora e Joazeiro (Estudos feitos à requisição da Inspectoria das Obras contra a Secca) Direcção do dr. Arrojado Lisbôa'. Mem. Inst. Oswaldo Cruz, Rio de Janeiro, 7 (1): 5-50, estampas 1-18.

'Contribuição para o estudo dos hematophagos. I. Sobre as partes bucaes dos nematoceros que sugam sangue'.

Mem. Inst. Oswaldo Cruz, Rio de Janeiro, 4 (1): 75-83.

'Segunda contribuição para o conhecimento das espécies brasileiras do gênero 'Simulium'. Rio de Janeiro, Instituto Oswaldo Cruz. Separata de Mem. do Inst. Oswaldo Cruz, Rio de Janeiro, 2 (2): 213-62, estampas 18-21 (em port. e alem.). 
Lutz, A. 1909

Lutz, A. 1903

Maia-Herzog, M. 1999

Maia-Herzog, M. 1991

Maia-Herzog, M.; Shelley, A. J. e Luna Dias, A. P. A. 1985

Marchon-Silva, V.; Lourenço de Oliveira, R.; Almeida, M. D. de; Silva-Vasconcelos, $\mathrm{A}$. da e Costa, J. 1996

Moraes, M. A. P. e Chaves, G. M. 1974

Pinto, C. 1940

Pinto, C. 1938

Pinto, C. 1932

Pinto, C. 1930

Shelley, A. J.; Luna Dias, A. P. A.; Maia-Herzog, M.; . Procunier, W. S. e Moraes, M. A. P out.-dez. 1987

Shelley, A. J.; Pinger, R. R. e Moraes, M. A. P. 1982

Sixto Coscarón 1991
'Contribuição para o conhecimento das espécies brasileiras do gênero 'Simulium'. Rio de Janeiro, Instituto Oswaldo Cruz. Separata de Mem. do Inst. Oswaldo Cruz, Rio de Janeiro, 1 (2): 124-46 (em port. e alem.).

'Nota preliminar sobre os insectos sugadores de sangue observados no Estado de São Paulo e Rio de Janeiro'. O Brazil-Medico, Rio de Janeiro, 17 (29): 281-2.

A dispersão da oncocercose no Brasil. Tese de doutoramento não publicada, Rio de Janeiro, Instituto Oswaldo Cruz, $\mathrm{V}+\mathrm{p}$.

Estudos comparativos de Simulium (Psaroniocompsa) incrustatum e Simulium (Psaroniocompsa) yarzabali (Diptera: Simuliidae). Dissertação de mestrado, Rio de Janeiro, Instituto de Biologia, Universidade Federal Rural do Rio de Janeiro, $\mathrm{V}+68 \mathrm{p}$.

'Simulium hirtipupa Lutz, 1910 (Dipetra: Simuliidae) - Descrição dos adultos e larva e redescrição da pupa'.

Mem. Inst. Oswaldo Cruz, 80 (4): 483-90.

'The type specimes of mosquitoes (Diptera, Culicidae) deposited in the entomological collection of the Instituto Oswaldo Cruz, Rio de Janeiro, Brazil'. Mem. Inst. Oswaldo Cruz, 91 (4): 471-8.

'Um caso de oncocercose no território de Roraima, Brasil'.

Rev. Inst. Med. Trop. São Paulo, 16: 110-3.

'Simuliidae da região neotropical'. Arq. Zool. São Paulo, 1 (12): 383-85.

Zôo-parasitas de interesse médico e veterinário. Rio de Janeiro, 137-139.

'Simuliidae da America Central e do Sul'.

$7^{a}$ Reunión de la Sociedad Argentina de Patología Regional Del Norte (1931),

7: 661-763, 41 figs $+\mathrm{i}$ (corrigenda).

'Arthrópodes parasitos e transmissores de doenças'.

Tratado de Parasitologia, vol. IV, 2: 397-845.

'Identification of vector species (Diptera: Simuliidae) of human onchocerciasis in the Amazonia focus of Brazil and Venezuela'.

Mem. Inst. Oswaldo Cruz, Rio de Janeiro, vol. 82 (4): 461-5.

'The taxonomy, biology and medical importance of Simulium amazonicum Goeldi, 1905 (Diptera: Simuliidae) with a review of related species'.

Bull. Br. Mus. Nat. Hist. (Ent.)' 44 (1): 1-29.

'Fauna de agua dulce de la República Argentina'. Insect Diptera, vol. 38, fasc. 2, Simuliitae, Fundación para la Educaión, la Ciencia y la Cultura, Buenos Aires, 431, Moreno. 
Vulcano, M. A. 1967

Vulcano, M. A. 1962

Vulcano, M. A. 1959

Vulcano, M. A. e D’Andretta Jr., C. 1958

Vulcano, M. A. e D’Andretta Jr., C. 1952

Vulcano, M. A. e D'Andretta Jr., C. 1949

Vulcano, M. A. e D'Andretta Jr., C. 1947

Vulcano, M. A. e D'Andretta Jr., C. 1947

Vulcano, M. A. e D'Andretta Jr., C. 1945
'A catalogue of the Diptera of the Americas South of the United States. 16. Family Simuliidae'. São Paulo, Dept. Zool. Secret. Aric., 44p.

'Simulídeos da serra da Bocaina. Observações bionômicas, Revalidação de Simulium brevifurcatum Lutz e descrição de uma espécie nova, Simulium anamariae (Diptera, Simuliidae)'.

Papéis Avulsos Dep. Zool. São Paulo, 15: 172-239.

'Descrição do alótipo de Simulium pruinosum Lutz, 1910 e caracteres adicionais da fêmea'.

Mem. do Inst. Oswaldo Cruz, Rio de Janeiro, 57 (1): 33-43, estampas 1-10.

'Redescrição do Simulium rubrithorax Lutz, 1909, e descrição do alotipo macho (Diptera, Simuliidae)'. Papéis Avulsos do Dept. de Zoologia - Secretaria da Agricultura. São Paulo, 13 (18): 227-40.

'Espécies neotropicais da família Simuliidae (Diptera Nematocera). VII. Redescrição de Lutzsimulium pernigrum (Lutz, 1910), N. Comb. e considerações sôbre o gênero'. Papéis Avulsos do Dept. de Zoologia Secretaria da Agricultura. São Paulo, X (18): 307-24.

'Espécies neotropicais da família Simuliidade Schiner (Diptera-Nematocera). V. Redescrição do Simulium auristriatum Lutz, 1910 e Simulium

(Eusimulium) venustum infuscata, Lutz, 1909 var. inquirendae'. Ver. Brasil. Biol., 9 (1): 55-66.

'Redescrição de Gigantodax wrighti (Vargas, Martinez e Diaz, 1944)

Diptera Simuliidae'. Papéis Avulsos do Dept. de Zoologia - Secretaria da Agricultura. São Paulo, VIII (2): 23-37.

'Espécies neotropicais da família Simuliidae Schiner (Diptera-Nematocera). III. Simulium botulibranchium Lutz, 1910 e Simulium travassosi n. sp.'.

Papéis Avulsos do Dept. de Zoologia - Secretaria da Agricultura. São Paulo, VIII (13): 146-80.

'As espécies neotropicais da família Simuliidae Schiner, 1864

(Diptera-Nematocera). I. Simulium (Eusimulium) orbitale Lutz, 1910,

Simulium (Eusimulium) pintoi n. sp. E Simulium nigrimanum Macquart, 1837, sp. Inquirendae'. Mem. Inst. Oswaldo Cruz, 43 (1): 85-152. 